\title{
Detection of Above the Limb Cosmic Rays in the Optical Cherenkov Regime Using Sub-Orbital and Orbital Instruments
}

\author{
A. L. Cummings, ${ }^{a, b, *}$ R. Aloisio, ${ }^{a, b}$ J. Eser ${ }^{c}$ and J. F. Krizmanic ${ }^{d, e}$ \\ ${ }^{a}$ Gran Sasso Science Institute (GSSI), \\ L'Aquila, AQ 67100, Italy \\ ${ }^{b}$ INFN-Laboratori Nazionali del Gran Sasso, \\ Assergi, AQ 67100, Italy \\ ${ }^{c}$ Department of Astronomy \& Astrophysics, University of Chicago, \\ Chicago, IL 60637, USA \\ ${ }^{d}$ CRESST/NASA Goddard Space Flight Center, \\ Greenbelt, MD 20771, USA \\ ${ }^{e}$ University of Maryland, Baltimore County, \\ Baltimore, MD 21250, USA \\ E-mail: austinlee.cummings@gssi.it
}

\begin{abstract}
Many upcoming experiments seek to observe high energy cosmic events while observing from either sub-orbital or orbital altitudes, using the Earth atmosphere as an extremely large instrumental volume, thereby increasing the geometric acceptance of ground-based instruments in addition to supplying uniform exposure in both hemispheres. In particular, the planned Extreme Universe Space Observatory aboard a Super Pressure Balloon-2 (EUSO-SPB2) and future Probe Of Extreme Multi-Messenger Astrophysics (POEMMA) experiments will both utilize UV sensitive optical cameras with nanosecond time resolution and observe near the Earth's limb to attempt to capture the Cherenkov emission produced by upwards going extensive air showers (EASs) sourced from high energy cosmic neutrino interactions in the Earth. In addition, these Cherenkov cameras also have coverage above the Earth limb, allowing access to the Cherenkov emission produced by cosmic rays skimming the Earth atmosphere at high altitudes.

We show that sub-orbital and orbital based optical Cherenkov telescopes are quite sensitive to the above the limb cosmic ray signal, in particular the sub-orbital case being sensitive down to $\mathrm{PeV}$ scale energies, allowing for high event rates even for short observation periods. We argue that because the properties of the arriving Cherenkov photons are similar for neutrino induced EASs and those induced by above the limb cosmic rays, the latter provides an excellent benchmark for qualifying the technique for neutrino observation with a well understood and guaranteed signal.
\end{abstract}

$37^{\text {th }}$ International Cosmic Ray Conference (ICRC 2021)

July 12 th - 23rd, 2021

Online - Berlin, Germany

\footnotetext{
${ }^{*}$ Presenter
} 


\section{Introduction}

Observing the complementary astrophysical fluxes of high-energy radiation is the primary goal of Multi-Messenger Astronomy (MMA). The ability to be able to measure various highenergy particles (cosmic rays, gamma rays and neutrinos) reliably and in a timely manner is critical to achieving MMA goals. The observation of the Earth atmosphere from sub-orbital or orbital altitudes in particular highlights a method for expanding the exposure of ground-based instruments.

The planned Extreme Universe Space Observatory aboard a Super Pressure Balloon II (EUSOSPB2) [1] and future Probe Of Extreme Multi-Messenger Astrophysics (POEMMA) [2] missions utilize this concept from sub-orbital and orbital altitudes, respectively, by employing fast imaging (20 ns), Schimdt Optical Cherenkov telescopes to monitor near the Earth limb to capture the bright, beamed Cherenkov emission from Earth-skimming neutrino events.

In principle, cosmic rays with trajectories from above the limb can interact with Earth's atmosphere and produce an EAS with a resulting optical Cherenkov signal strong enough to be experimentally detectable, much like the Earth-skimming neutrino events. Both the EUSOSPB2 and POEMMA missions are designed such that the onboard Cherenkov camera can provide additional coverage above the Earth's limb, allowing for detection of these cosmic ray events.

Above-the-limb cosmic rays are an interesting signal to target with these Cherenkov telescopes for several reasons: 1) Cosmic rays are characterized by significantly higher fluxes than astrophysical neutrinos above PeV energies [3], leading to much larger expected event rates [4]. 2) The optical Cherenkov signal produced by above-the-limb cosmic rays has nearly identical properties to the emission produced by Earth-skimming neutrino events. Given their expectedly high event rate, the above-the-limb cosmic rays can provide a consistent benchmark to test the different components of the telescope (i.e. optics, electronics and triggers) directly in flight, thereby providing an in-situ determination of detector performance and verifying the detection method. 3) The Cherenkov intensity generated by the EAS is linearly proportional to the primary energy of the cosmic ray, and the high altitude development of the EAS ensures smaller angular scales, both of which lead to better reconstruction capabilities. 4) Above-the-limb cosmic rays have already been observed by the ANITA experiment $[5,6]$. As the energy threshold for radio observation is significantly higher than that for Cherenkov detection, this provides further motivation for a large event rate detection.

\section{Cherenkov Simulation}

Using the framework of the custom Cherenkov simulation developed in [7] for Earth-skimming neutrinos, the properties of the optical Cherenkov emission from an above-the-limb cosmic ray induced EAS can be generated for a given shower geometry.

Figure 1 shows a sketch of the geometry for observing the above-the-limb cosmic rays and typical atmospheric grammage profiles for different EAS trajectories in the case of balloon-based observation. By taking a representative shower with $X_{\max }=700 \mathrm{~g} \mathrm{~cm}^{-2}$, corresponding to the case of a primary $100 \mathrm{PeV}$ proton, we observe that the altitude of maximum shower development has a minimum of $\sim 20 \mathrm{~km}$ (which is also true for observation from orbital altitudes [4]), which increases with increasing $\theta_{d}$ (see figure 1), indicating the need to carefully account for shower development at high altitudes, where the atmosphere is rarefied. 

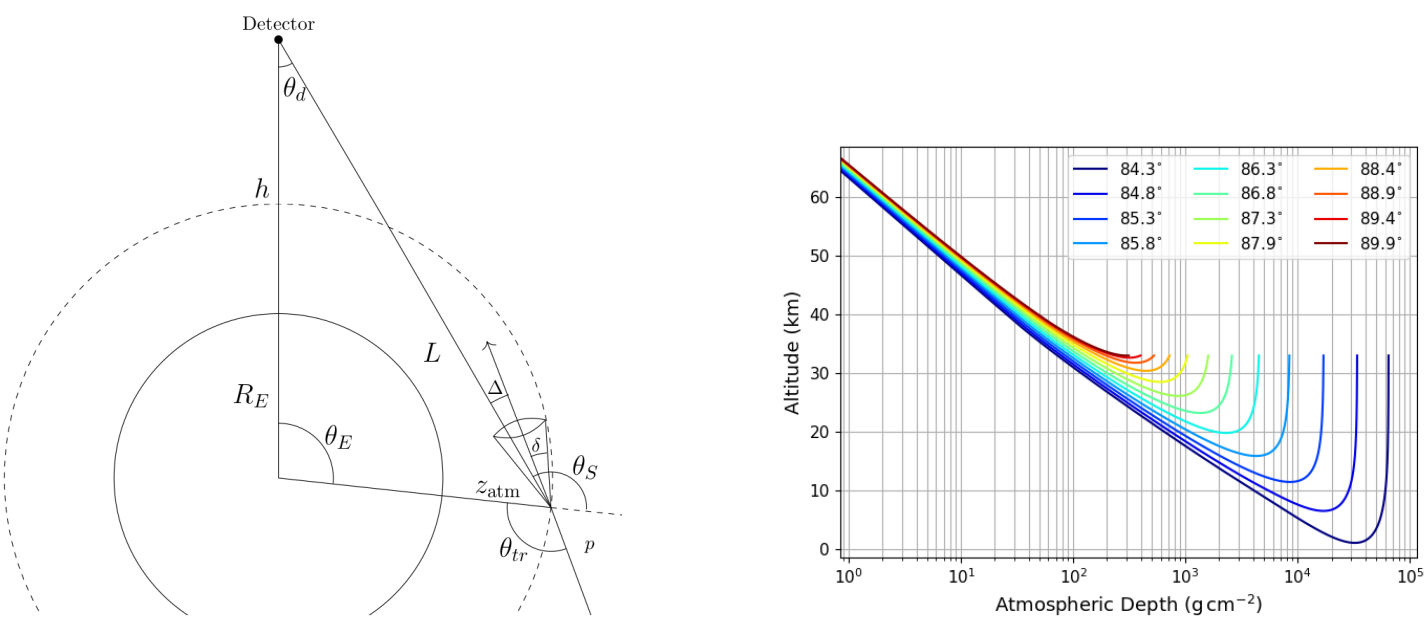

Figure 1: [Left panel] Sketch of the geometry for observation of the optical Cherenkov emission from above-the-limb cosmic ray induced EAS from a space-based instrument. [Right panel] Cumulative slant depth as a function of altitude and detector viewing angle $\theta_{d}$ from nadir, as measured from $33 \mathrm{~km}$ altitude.

Specifically, for altitudes above $25 \mathrm{~km}$, the scales corresponding to the gyration radius on the geomagnetic field of an electron at the Cherenkov threshold and its radiation length in air are comparable [8], indicating the need to consider the effect of the geomagnetic deflection of electrons and positrons in the EAS on the resultant Cherenkov emission. The deflection angle of the generating $e^{ \pm}$was calculated, taking into account the particle's energy, charge, and orientation with respect to the incident Earth magnetic field and applied to the Cherenkov photons reaching the detection plane. To bound the maximum and minimum effects of the magnetic field, we consider only the orientation in which the field is applied perpendicular to the initial cosmic ray trajectory, and measure the resultant Cherenkov photon distribution along the axes parallel and perpendicular to the field on the detection plane. An example fit of the Cherenkov spatial distribution with and without an applied geomagnetic field is shown in the left panel of figure 2. The effect of the magnetic field is to spread Cherenkov photons near the shower axis angle further away in the direction perpendicular to the field, thus strengthening the intensity of the tails. Across nearly all shower geometries observable by an orbital or suborbital detector, the central Cherenkov intensity remains within a modest factor of 2 between the case where the geomagnetic field is applied and the case where no such field is applied.

Additionally, we expect that cosmic rays interacting in the Earth atmosphere can deposit most of their energy into the ensuing EAS, resulting in extremely bright Cherenkov emission, which can, in principle, be visible far off the shower axis, leading to large geometric apertures. However, from downwards going EAS, it is known that the Cherenkov photons which arrive on the detection plane have larger spreads in arrival time the further off shower axis they are recorded, thereby decreasing the signal to noise ratio within the instrumental integration time and reducing the sensitivity [9]. We have considered the time spreading effects of the Cherenkov photons by incorporating the electron time-lag distributions as given by [10] and taking into account the geometry dependent photon propagation time through the atmosphere. The $90 \%$ time spread (the $\Delta t$ required to capture $90 \%$ of the arriving photons) for the example shower shown in the left panel of figure 2 is shown in 

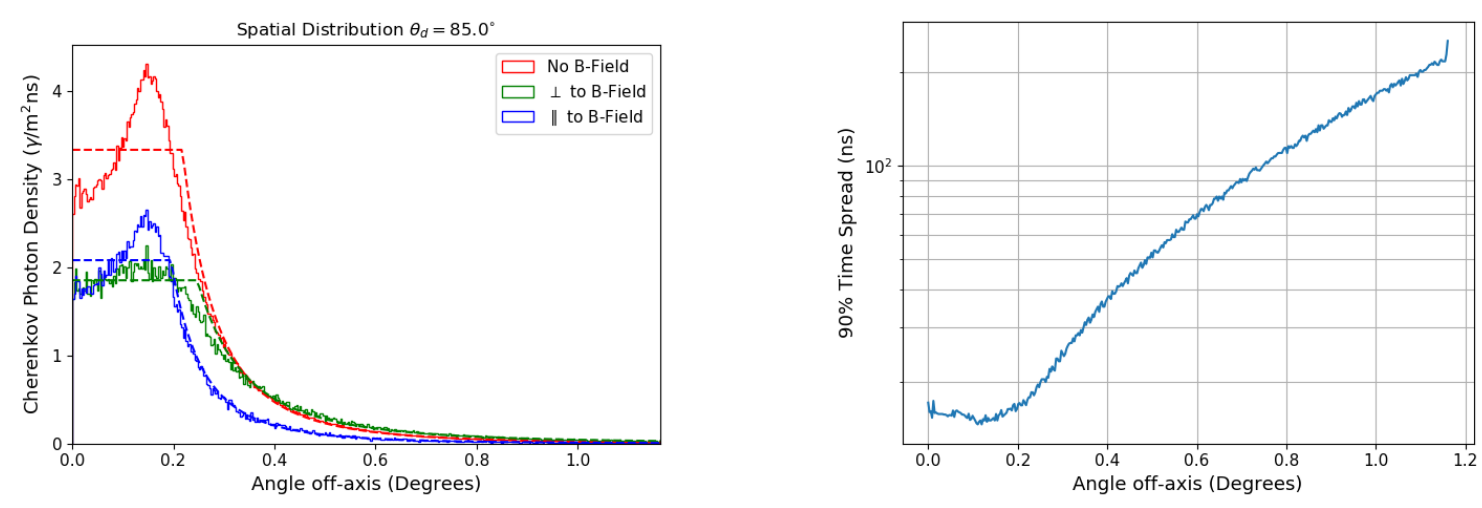

Figure 2: [Left panel] Cherenkov spatial distributions of a $100 \mathrm{PeV}$ proton shower as observed from $33 \mathrm{~km}$ with $\theta_{d}=85^{\circ}$. The Cherenkov photon distributions are computed in the case where no geomagnetic field is applied (red curve) and where a $50 \mu \mathrm{T}$ magnetic field is applied perpendicular to the shower propagation direction. The green and blue curves show the components of the spatial distribution measured along the axes perpendicular and parallel to the magnetic field, respectively. The dashed lines show the result of a 5 parameter fit. [Right panel] 90\% time spread of the arriving Cherenkov photons for the same shower.

the right panel of the same figure. As observed, the time spread of the arriving photons far from shower axis can grow considerably larger than the nominal spread near the Cherenkov ring, often reaching up to a microsecond in width. The Cherenkov cameras of EUSO-SPB2 and POEMMA have integration times of $10 \mathrm{~ns}$ and $20 \mathrm{~ns}$, respectively, so including this effect is fundamental in modeling the sensitivity to these Cherenkov signals.

\subsection{Monte Carlo Calculation of Aperture}

Using the custom Cherenkov simulation described in section 2, we create a lookup table of the properties of the arriving Cherenkov photons as measured on the detection plane. The geometric parameter space within which the lookup table is generated considers i) the trajectory through the atmosphere, defined by the angle $\theta_{d}$ (see left panel of figure 1) and ii) the first interaction depth of the cosmic ray $X_{0}$.

To calculate the geometric aperture of the considered telescopes to the above-the-limb cosmic ray flux, we use a Monte Carlo calculation, where, for each simulated EAS, the trajectory starting point is sampled isotropically from the top of the Earth atmosphere (in the US standard atmosphere, $112 \mathrm{~km}$ ) and the trajectory direction is also sampled isotropically.

The first interaction depth $X_{0}$ for a generated EAS is sampled from an exponential distribution with mean $\lambda$, given by:

$$
\lambda(E)=A m_{N} / \sigma(E)
$$

where $A=14.1$ is the average atomic mass of air, $m_{N}$ is the mass of a nucleon, and $\sigma(E)$ is the proton-air interaction cross section as a function of primary energy, as given in [8].

Using the sampled $\theta_{d}$ and $X_{0}$, we interpolate the appropriate parameters of the Cherenkov distribution at the instrument from the generated lookup table, and scale the overall intensity by a factor $E / 100 \mathrm{PeV}$ to account for the energy with which the lookup table was generated. 

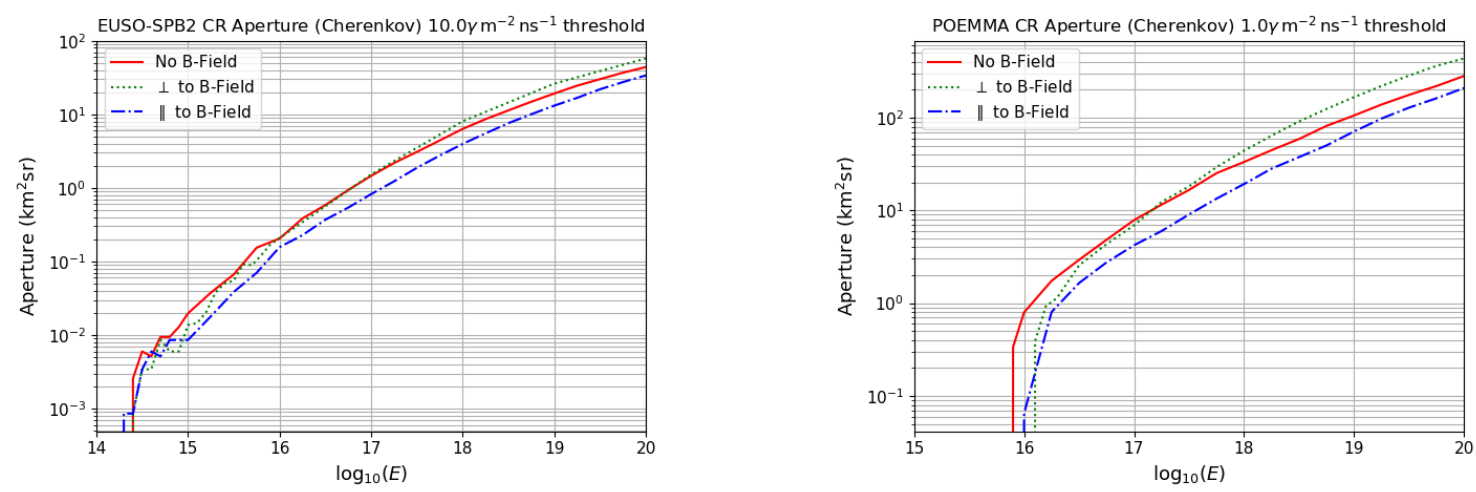

Figure 3: Geometric aperture to above-the-limb cosmic rays as a function of primary energy for the EUSOSPB2 [left panel] and POEMMA [right panel] detectors.

We consider an event to be accepted if the Cherenkov intensity at aperture surpasses $200 \mathrm{\gamma} / \mathrm{m}^{2}$ for EUSO-SPB2 or $20 \gamma / \mathrm{m}^{2}$ for POEMMA. These thresholds are calculated considering primarily the dark-sky airglow background, as outlined in [7, 11]. To account for the effect of the time spreading of the arriving photons, this expected threshold is divided by the minimum electronics integration time, which we consider to be nominally $20 \mathrm{~ns}$.

For every primary cosmic ray energy simulated, we consider $10^{5}$ shower starting points and $10^{5}$ shower trajectories for each sampled starting point. The geometric aperture is then calculated as:

$$
\begin{aligned}
\langle A \Omega\rangle(E) & =\pi S \frac{N_{\text {accepted }}(E)}{N_{\text {simulated }}(E)} \\
S & =\int_{0}^{2 \pi} \int_{\theta_{E 1}}^{\theta_{E 2}}\left(R_{E}+z_{\text {atm }}\right)^{2} \sin \theta_{E} d \theta_{E} d \phi
\end{aligned}
$$

where $S$ is the area of the disk visible to the instrument $[4,12,13]$. To bound the effects of the magnetic field, we perform this calculation for 3 scenarios: i) No applied geomagnetic field ii) Maximally applied geomagnetic field, assuming all observations made perpendicular to the field iii) Maximally applied field, assuming all observations made parallel to the field. In Figure 3, we plot the geometric apertures of the EUSO-SPB2 (left panel) and POEMMA (right panel) experiments to the above-the-limb cosmic rays.

Figure 3 demonstrates that, as expected, the EUSO-SPB2 instrument has increased exposure for energies below $10 \mathrm{PeV}$ due to being closer to the shower development. At higher energies, the sensitivity of the POEMMA instrument begins to dominate, becoming roughly an order of magnitude larger than that of EUSO-SPB2 at $10^{20} \mathrm{eV}$. The effect of the geomagnetic field is modest, resulting in a maximum difference of $\sim 2$ between the affected and unaffected cases.

In figure 4, we plot the normalized distribution of the arrival angle $\theta_{d}$ for the accepted events using the Monte Carlo methodology. Few events are accepted above $90^{\circ}$ and $68.35^{\circ}$ for EUSOSPB2 and POEMMA, respectively, which corresponds to trajectories with a total slant depth of $\sim 300 \mathrm{~g} \mathrm{~cm}^{-2}$. Higher energy primaries are also more observable near the limb, as the higher 

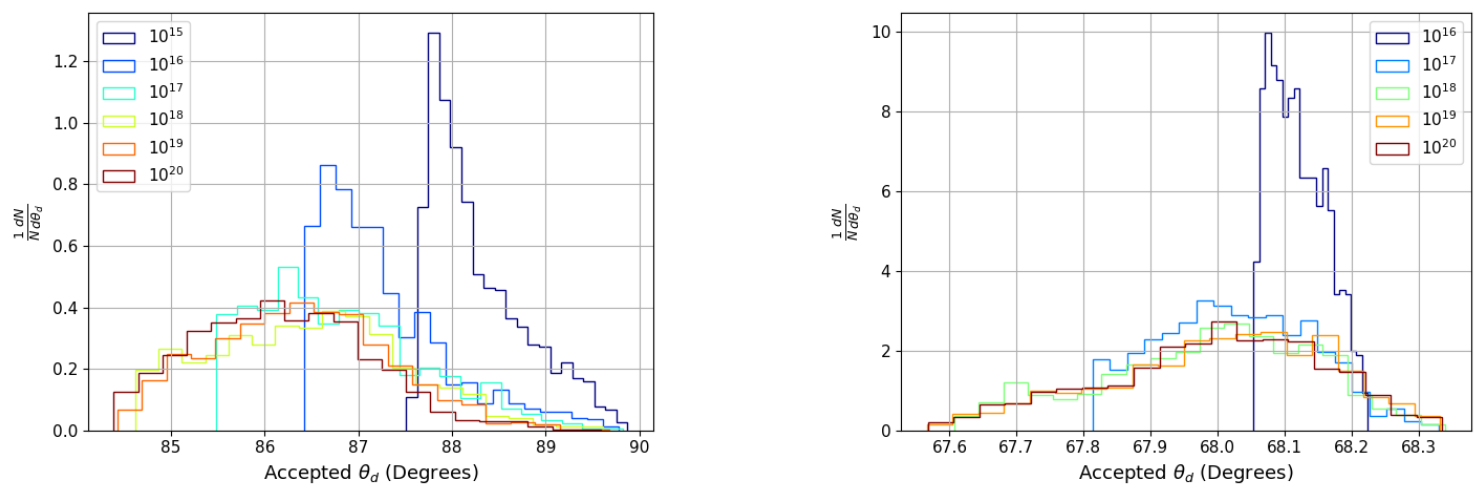

Figure 4: Normalized distribution of arrival angle $\theta_{d}$ for accepted above-the-limb cosmic rays for different primary energies as measured with the EUSO-SPB2 instrument [left panel] and POEMMA instrument [right panel].

intensity of the generated Cherenkov emission is able to compete with the strong atmospheric extinction.

\section{Discussion}

Given a flux of cosmic rays $\Phi_{C R}(E)$ and the geometric aperture $\langle A \Omega\rangle(E)$ as obtained via the Monte Carlo technique above, we calculate the cumulative event rate above a primary energy $E$ as:

$$
N=\iint_{E}^{\infty}\langle A \Omega\rangle(E) \Phi_{C R}(E) d E d t
$$

We use the cosmic ray flux $\Phi_{C R}(E)$, as described by the combined, all particle energy spectra from the Tibet-ASg, KASCADE-Grande, and Pierre-Auger experiments across the range $\left(10^{14} \mathrm{eV}, 10^{20} \mathrm{eV}\right)$ given in [8]. In figure 5, we plot the estimated event rate per hour of experimental live time of above-the-limb cosmic rays as a function of minimum energy in for both EUSO-SPB2 and POEMMA. For both orbital and sub-orbital Cherenkov telescopes, the expected event rate is very large, being hundreds of events per hour of live time above energies of less than a PeV in the sub-orbital case and $10 \mathrm{PeV}$ in the orbital case.

The properties of the optical Cherenkov emission from the above-the-limb cosmic ray events (spatial, wavelength, angular, and timing distributions of arriving photons) are extremely similar to those from neutrino induced signals, despite the significant differences in the shower geometries [7]. Considering the large estimated event rates projected in figure 5, the above-the-limb cosmic rays represent a guaranteed in-flight test source for both orbital and sub-orbital optical Cherenkov telescopes. While Earth-skimming neutrino events from the diffuse cosmogenic neutrino flux are expected to be rare (although, in the case of an astrophysical transient follow-up, the probability of observation can be significantly increased, see [14]), the high event rate of the above-the-limb cosmic rays allows for validation of the optical Cherenkov detection technique for upward going EAS. These observations will help quantify optical performance, trigger algorithms, and other detection strategies such that the telescope is optimized for neutrino detection. 

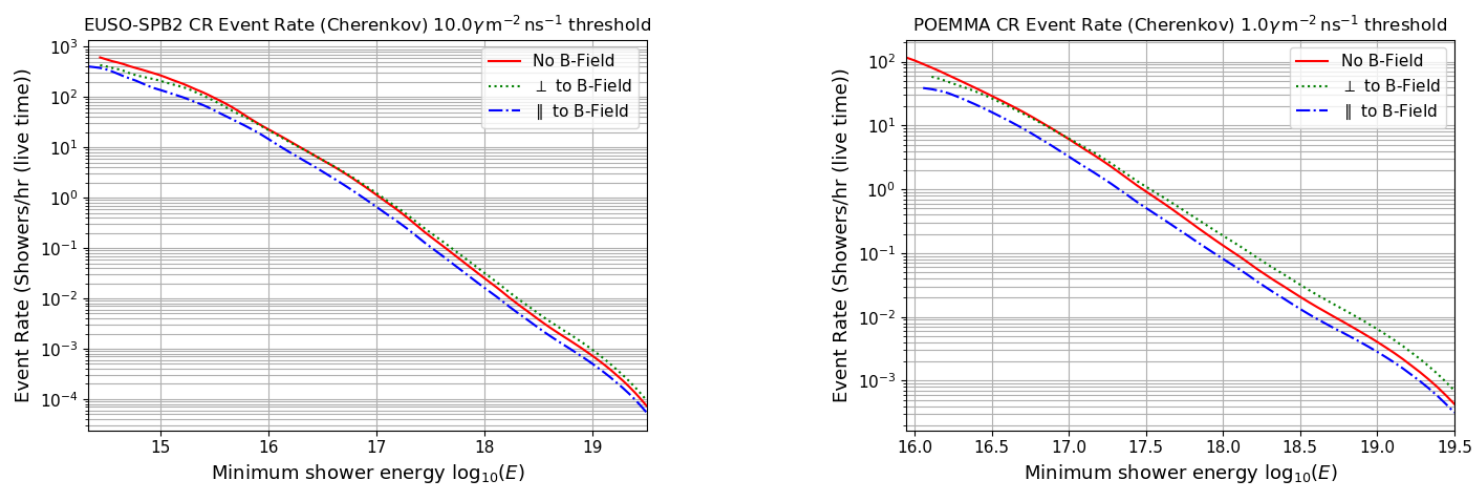

Figure 5: Integrated expected event rate (events measured above given energy $E$ ) for above-the-limb UHECR events for the EUSO-SPB2 [left panel] and POEMMA [right panel] instruments. Event rate is given per hour of live time (instrument duty cycle for each not taken into account).

Figure 4 demonstrates that there is limited acceptance of CR near the Earth limb, indicating a nontrivial event rate. Because atmospheric refraction of light near the limb can reach up to $\sim 1^{\circ}$, it is likely that these signals can be reconstructed as originating from below the limb, thereby being mistaken as neutrino-induced events [15]. To contend with this effect, future neutrino searches may need to be restricted to at least $1^{\circ}$ below the limb to avoid potential false positive signatures.

The above-the-limb cosmic rays are good candidates for energy and directional reconstruction for several reasons: (i) the signal intensity is approximately linearly proportional to the primary cosmic ray energy (ii) the angular acceptance is energy dependent (iii) the overall angular scales are small due to the high altitude development, leading to exceptional angular resolution of the trajectory of the EAS (iv) the high number of events allows for large statistical groupings. The reconstruction methods used to identify the cosmic rays will be studied and improved using the collected flight data, hopefully to be implemented also for neutrino reconstruction.

Mass composition measurements of the above-the-limb cosmic rays using the optical Cherenkov detection technique may also be possible using the strategies developed for ground-based telescopes: (i) The observation and discrimination of the Cherenkov emission generated by muons within the EAS, which helps to clarify the nature of the primary particle [16] and (ii) Multiple observations within the effective Cherenkov angle of the resulting emission, which allows for an estimation of $X_{\max }$ on an EAS-by-EAS basis [17].

\section{References}

[1] JEM-EUSO, POEMMA collaboration, The Extreme Universe Space Observatory on a Super-Pressure Balloon II Mission, PoS ICRC2019 (2020) 466 [1909. 12835].

[2] A. Olinto et al., The POEMMA (Probe of Extreme Multi-Messenger Astrophysics) Observatory, 2012.07945.

[3] R. Aloisio, P. Blasi, I. De Mitri and S. Petrera, Selected Topics in Cosmic Ray Physics, pp. 1-95 (2018), DOI [1707.06147]. 
[4] A. Cummings, R. Aloisio, J. Eser and J. Krizmanic, Modeling the Optical Cherenkov Signals by Cosmic Ray Extensive Air Showers Directly Observed from Sub-Orbital and Orbital Altitudes, 2105.03255.

[5] ANITA collaboration, Observation of Ultra-high-energy Cosmic Rays with the ANITA Balloon-borne Radio Interferometer, Phys. Rev. Lett. 105 (2010) 151101 [1005. 0035].

[6] H. Schoorlemmer et al., Energy and Flux Measurements of Ultra-High Energy Cosmic Rays Observed During the First ANITA Flight, Astropart. Phys. 77 (2016) 32 [1506. 05396].

[7] A.L. Cummings, R. Aloisio and J.F. Krizmanic, Modeling of the tau and muon neutrino-induced optical cherenkov signals from upward-moving extensive air showers, Physical Review D 103 (2021) .

[8] T.K. Gaisser, R. Engel and E. Resconi, Cosmic Rays and Particle Physics: 2nd Edition, Cambridge University Press (6, 2016).

[9] A.N. Otte, Studies of an air-shower imaging system for the detection of ultrahigh-energy neutrinos, Phys. Rev. D99 (2019) 083012 [1811.09287].

[10] S. Lafebre, R. Engel, H. Falcke, J. Horandel, T. Huege, J. Kuijpers et al., Universality of electron-positron distributions in extensive air showers, Astropart. Phys. 31 (2009) 243 [0902.0548].

[11] M.H. Reno, J.F. Krizmanic and T.M. Venters, Cosmic tau neutrino detection via Cherenkov signals from air showers from Earth-emerging taus, Phys. Rev. D100 (2019) 063010 [1902.11287].

[12] C. Crannell and J. Ormes, Geometrical - factor determination using a monte carlo approach, Nuclear Instruments and Methods 94 (1971) 179.

[13] J. Sullivan, Geometric factor and directional response of single and multi-element particle telescopes, Nuclear Instruments and Methods 95 (1971) 5.

[14] T.M. Venters, M.H. Reno, J.F. Krizmanic, L.A. Anchordoqui, C. Guépin and A.V. Olinto, Poemma's target-of-opportunity sensitivity to cosmic neutrino transient sources, Physical Review D 102 (2020) .

[15] W.P. Chu, Calculations of atmospheric refraction for spacecraft remote-sensing applications, Appl. Opt. 22 (1983) 721.

[16] A. Neronov, D.V. Semikoz, I. Vovk and R. Mirzoyan, Cosmic-ray composition measurements and cosmic ray background-free-ray observations with cherenkov telescopes, Physical Review D 94 (2016) .

[17] N. Budnev et al., TAIGA the Tunka Advanced Instrument for cosmic ray physics and Gamma Astronomy - Present status and perspectives, Journal of Instrumentation 9 (2014) C09021. 